\title{
Levinson's Theorem and the Nonlocal Saito Potential
}

\author{
S.B. Qadri \\ Department of Physics, Ohio State University \\ Columbus, Ohio 43210, USA \\ Naval Research Laboratory, Washington, DC 20375 USA \\ Tel: 1-202-404-4129 E-mail: qadri@anvil.nrl.navy.mil \\ B. Mulligan \\ Department of Physics, Ohio State University \\ Columbus, Ohio 43210, USA \\ M.F. Mahmood \& J.Y. Al-Khal \\ Department of Mathematics, Howard University Washington \\ DC 20059, USA
}

\begin{abstract}
We consider Levinson's theorem for the nonlocal Saito potential. We find that the phase of the Jost function gives a correct result for the zero-energy phase shift of $\pi$ associated with the additional node in the scattering wavefunction. This analysis takes into account the zero-energy continuum bound state of the potential. A comparison is given with previous results which do not consider the possibility that such a state is present.
\end{abstract}

Keywords: Levinson's Theorem, Nonlocal potentials, Continuum bound states, Spurious states, Jost functions, Fredholm determinants, Definition of the phase shift

\section{Introduction}

In nucleon-nucleus and nucleus-nucleus scattering, antisymmetrization results in nonlocal effective two-body interactions which may be very tedious to obtain and quite elaborate in their final form (Special Supplement of the Progress of Theoretical Physics, 1977). It was first suggested by Saito $(1968,1969)$ that such complicated nonlocal kernels could be approximated by simplied nonlocal kernels which retained the fundamental features associated with the Pauli exclusion principle. In particular, Saito showed that his approach could produce the extra nodes in the two-body relative scattering wave-function required for orthogonality of that wavefunction with respect to already occupied single particle states.

Because the Saito model has a variety of applications, it has been studied extensively. One aspect of such studies has been to understand how the Saito model produces extra nodes in the scattering wavefunction. It is now known (B. Bagchi, 1978) that the mechanism by which the Saito model achieves an extra node (or nodes) is that of using a kernel with a continuum bound state (A. Martin, 1958; B. Mulligan, 1976) at zero energy. When placed at zero energy, a continuum bound state affects the scattering spectrum at positive energies only through the presence of the additional node. This additional node can be explicitly demonstrated (B. Bagchi, 1980) as forcing the scattering wavefunction to be orthogonal to the wavefunction of the continuum bound state. Thus Saito's approach can orthogonalize a scattering wavefunction with respect to any state or, by including several continuum bound states at zero energy, with respect to any set of states. This leads to a scattering wavefunction with the required number of extra nodes.

When studying the nodal structure of a scattering wavefunction, it is customary to relate the nodes in the zero-energy wavefunction to the zero-energy phase shift. Such a relationship was first established in the case of a local potential by Levinson (N. Levinson, 1949). The generalization of Levinson's theorem to nonlocal potentials has been investigated by many authors (B. Mulligan and S.B. Qadri). However, attempts to discuss Levinson's theorem in the context of the Saito model (N.J. Englefield, 1974; W. Glockle, 1976) have failed to explain the mechanism by which the extra nodes are produced. In particular, both Refs. (N.J. Englefield, 1974) and (W. Glockle, 1976) explicitly exclude the effects on Levinson's theorem resulting from a zero-energy continuum bound state. As has been discussed in detail by Newton 
(R.G. Newton, 1960), the question of a boundstate at zero energy requires special attention. Let us focus, for example, on the $\ell=0$ radial equation. As Newton shows (R.G. Newton, 1960; 1977, P1348) for a local potential this radial equation cannot have a bound state at zero energy although a half-bound state is possible. On the other hand, a nonlocal potential can have many zero-energy bound states (R.G. Newton, 1977, P1582). In any such circumstance, a slight adjustment of the potential parameters can move the bound states into the continuum. Thus, we easily recognize a bound state of this kind as a zero-energy continuum bound state.

In the present paper, we explain the relationship between Levinson's theorem and the extra nodes generated in Saito's model. We will restrict our considerations to the $\ell=0$ case and to the particular example from the class of nonlocal potentials associated with the Saito model which has become known as the Saito potential (S. Okai, 1972). In the following sections, first we describe the radial equation for $\ell=0$ case and discuss the integral equations associated with various solutions and their corresponding Fredholm determinants. By calculating the Fredholm determinants for Saito potential, we show the origin of the extra node by contour integration. This work will have impact in nuclear scattering theory for nonlocal potentials.

\section{The saito potential and the jost function}

The $\ell=0$ radial equation with the Saito potential is

$$
\left(\frac{d^{2}}{d r^{2}}+k^{2}\right) u(k, r)=\xi(r) \int_{0}^{\infty} \xi(s) \frac{d^{2}}{d s^{2}} u(k, s) d s
$$

where

$$
\xi(r)=\left(4 a^{3}\right)^{1 / 2} r e^{-\alpha r}
$$

The procedure we use for discussing Levinson's theorem for the Saito potential is based on the Jost function $\mathfrak{L}^{+}(k)$, and follows the approach of Swan (P. Swan, 1955). The Jost function is defined as the Jost solution evaluated at $r=0$.

$$
\mathfrak{L}^{+}(k)=\left.f^{+}(k, r)\right|_{r=0}
$$

Swan noted that on the positive imaginary axis in the complex k plane the Jost function $\mathfrak{L}^{+}(k)$ for a local potential has a zero associated with each bound state (H.M. Nussenzveig, 1972). Thus Swan was able to make use of a theorem from complex analysis, known as the argument principle, which states that if a function is analytic inside and on a simple contour, except for a finite number of poles inside the contour, and if the function has no zeros on the contour, then the integral of the logarithmic derivative of that function around the contour is equal to $2 \pi i$ times the number of zeros inside the contour minus the number of poles inside the contour. For a central local potential it therefore is possible to construct an integral around a path in the upper half plane 3 such that this integral is $2 \pi i$ times the number of bound states of the potential. Since for a local potential the scattering phase shift is given by the negative of the phase of $\mathfrak{L}^{+}(k)$, Levinson's theorem follows immediately. As indicated by Swan, his procedure can be generalized for use with nonlocal potentials. For a symmetric nonlocal potential, the definition of the Jost function given by Eq. (3) can be applied without ambiguity (B. Bagchi, 1979, P1251). However, the Saito potential is not symmetric. Difficulties associated with the definition of the Jost function in the case of a nonsymmetric nonlocal potential have been discussed in Ref. (B. Bagchi, 1979, p1973). It is shown in Ref. (B. Bagchi, 1978) that in the case of the Saito potential the difficulties can be overcome. In this connection, we replace the definition of the Jost function given in Eq. (3) by one in terms of Fredholm determinants.

\section{Integral equations and fredholm determinants}

It was first demonstrated by Jost and Pais (R. Jost, 1951) that for a local potential the Jost function is related to Fredholm determinants of integral equations for solutions of the radial equation. The same situation has been demonstrated to be the case for a symmetric nonlocal potential (C.S. Warke, 1971; Y Singh, 1971; S.S. Ahmed, 1974). For a nonsymmetric nonlocal potential, the appropriate relation is discussed in Ref. (B. Bagchi, 1979, P1973).

The integral equations of interest are those for the physical solution, the regular solution, and the Jost solution. The physical solution of Eq. (1) satisfies the integral equation

$$
\psi^{+}(k, r)=\sin k r+\int_{0}^{\infty} G^{+}\left(k, r, r^{\prime}\right) \xi\left(r^{\prime}\right) d r^{\prime} \int_{0}^{\infty} \xi(s) \frac{d^{2}}{d s^{2}} \psi^{+}(k, s) d s
$$

Where

$$
G^{+}\left(k, r, r^{\prime}\right)=-k^{-1} e^{i k r>} \sin k r_{<}
$$

The regular solution of Eq. (1) satisfies the integral equation

$$
\varphi(k, r)=k^{-1} \sin k r+\int_{0}^{r} G\left(k, r, r^{\prime}\right) \xi\left(r^{\prime}\right) d r^{\prime} \int_{0}^{\infty} \xi(s) \frac{d^{2}}{d s^{2}} \varphi(k, s) d s
$$


where

$$
G\left(k, r, r^{\prime}\right)=k^{-1} \sin k\left(r-r^{\prime}\right)
$$

The integral equation for the Jost solution is

$$
f^{+}(k, r)=e^{i k r}-\int_{r}^{\infty} G\left(k, r, r^{\prime}\right) \xi\left(r^{\prime}\right) d r^{\prime} \int_{0}^{\infty} \xi(s) \frac{d^{2}}{d s^{2}} f^{+}(k, s) d s
$$

where $\mathrm{G}$ is given by Eq. (7).

The Fredholm determinants associated with the kernels of Eqs. (4), (6), and (8) are, respectively, $D^{+}(k), D(k)$, and $\triangle(k)$. It is shown in Ref. 3 that for the Saito potential $D(k)=\Delta(k)$. Thus for the Saito potential, the Jost function can be defined (B. Bagchi, 1979, P1251; P1973) as

$$
\mathfrak{L}^{+}(k)=\frac{D^{+}(k)}{D(k)}
$$

\section{Levinson's theorem for the saito potential}

The Fredholm determinants $D^{+}(k)$ and $D(k)$ for the Saito potential are given in Ref. (B. Bagchi, 1978). From the expressions for $D^{+}(k)$ and $D(k)$, it is evident that there is a continuum bound state at $k=0$. Using Eq. (9), the expression for $\mathfrak{L}^{+}(k)$ is

$$
\mathfrak{L}^{+}(k)=\frac{(k+\alpha+2 i \alpha)(k-\alpha+2 i \alpha)(k-i \alpha)^{2}}{(k+i \alpha)^{2}(k+i \sqrt{3} \alpha)(k-i \sqrt{3} \alpha)}
$$

In the upper half-plane, $\mathfrak{L}^{+}(k)$ has a double zero at $k=i \alpha$ and a pole at $k=i \sqrt{3} \alpha$. However, Eq. (10) contains no explicit information about the continuum bound state at $k=0$. In taking the ratio of $D^{+}(k)$ and $D(k)$ to form $\mathfrak{L}^{+}(k)$, the double zero of $D^{+}(k)$ at $k=0$ is canceled by the double zero of $D(k)$, leaving no zero of $\mathfrak{L}^{+}(k)$ at $k=0$. [Note that for a half bound state at $k=0$, a zero of $\mathfrak{L}^{+}(k)$ is required.]

Thus, as noted in Ref. (B. Mulligan, 1981), for a nonlocal potential the Jost function contains incomplete information. Nevertheless, it can still be used for a correct derivation of Levinson's theorem. It is demonstrated in Ref. (B. Bagchi, 1977) that if the phase shift is taken as the negative of the phase of $\mathfrak{L}^{+}(k)$, then Levinson's theorem as derived from $\mathfrak{L}^{+}(k)$ will yield a zero-energy phase shift of $\pi$ for each extra node in the zero-energy wavefunction due to a continuum bound state. It is shown in Ref. (B. Bagchi, 1978) that the zero-energy scattering wavefunction for the Saito potential has an extra node. Thus we would expect a derivation of Levinson's theorem using $\mathfrak{L}^{+}(k)$ to yield a zero-energy phase shift of $\pi$ for the Saito potential.

That this is the case can be easily demonstrated. Using the logarithmic derivative of $\mathfrak{L}^{+}(k)$, the argument principle for the contour $C$ of Fig. 1 yields

$$
\int_{C} \frac{\mathfrak{L}^{+}(k)^{\prime}}{\mathfrak{L}^{+}(k)} d k=\left.\ln \mathfrak{L}^{+}(k)\right|_{c}=2 \pi i
$$

Taking the phase shift to be defined as the negative of the phase of $\mathfrak{L}^{+}(k)$, that is, taking

$$
\mathfrak{L}^{+}(k)=\left|\mathfrak{L}^{+}(k)\right| e^{-i \delta(k)}
$$

and making use of the fact that $\delta(-k)=-\delta(k)$, Eq. (11) becomes

$$
2 i[\delta(0)-\delta(\infty)]=2 \pi i
$$

Thus we get

$$
\delta(0)-\delta(\infty)=\pi
$$

\section{Comparison with previous work}

The discussion of the properties of the Saito potential by Englefield and Shoukry (1974) is based upon a formulation of the problem in momentum space. Using this approach, Englefield and Shoukry conclude that the effect of the Saito potential in adding an extra node to the scattering wavefunction is to increase the zero-energy phase shift by $\pi$. However, they do not attribute this extra $\pi$ to a bound state at zero energy. Rather, they point out that their conclusions might need to be modified if a bound state at zero energy were to be included.

Glockle and Le Tourneux (1976) analyze the Saito potential by the more conventional method of contour integration in k space. The function which they select for forming a logarithmic derivative for the application of the argument principle yields the extra $\pi$ at $k=0$ in the phase shift due to the Saito potential. However, this is because the function which they choose [the function $D(k)$ defined on page 19 of Ref. (W. Glockle, 1976) happens to be constructed such that the double 
zero on the real $k$ axis due to the continuum bound state has been removed. Furthermore, they explicitly point out that they do not consider the possibility of a continuum bound state at $k=0$, and that their conclusions would have to be modified were such a state to be present. In particular, they point out (W. Glockle, 1976, P30) that, in the presence of a continuum bound state, an additional $\pi$ must be added.

\section{Conclusion}

In the present paper we have shown that the standard approach initiated by Swan (1981), when applied in the customary manner using the Jost function $\mathfrak{L}^{+}(k)$, gives results which are consistent with the extra node present in the scattering wavefunction for the Saito potential. We also reemphasize a conclusion presented in Ref. (B. Mulligan, 1981): Although a calculation of the phase shift at zero-energy using the Jost function gives a correct result in the presence of a continuum bound state, the Jost function does not itself have zeros on the real $\mathrm{k}$ axis associated with the continuum bound state. The Fredholm determinant $D^{+}(k)$, on the other hand, does have a pair of zeros on the real $k$ axis for each such state.

\section{Acknowledgments}

We thank Dr. B. Bagchi for several useful and stimulating discussions.

\section{References}

A. Martin. (1958). Nuovo Cimento, 7, 607.

B. Bagchi and B. Mulligan. (1979). Phys. Rev. C 20, 1973.

B. Bagchi, B. Mulligan, and S.B. Qadri. (1979). Phys. Rev. C 20, 1251.

B. Bagchi, B. Mulligan, and S.B. Qadri. (1978). Prog. Theor. Phys. 60, 765.

B. Bagchi, B. Mulligan, and T.O. Krause. (1980). Phys. Rev. 21, 1.

B. Bagchi, T.O. Krause, and B. Mulligan. (1977). Phys. Rev. C 15, 1623. 9.

B.Mulligan and S.B. Qadri. submitted to Phys. Rev. C. This paper discusses and analyzes much of the literature on the subject.

B. Mulligan, and S.B. Qadri. (1981). Phys. Rev. C 20, 874.

B. Mulligan, L.G. Arnold, B. Bagchi, and T.O. Krause. (1976). Phys. Rev. C, 13, 2131.

C.S. Warke and R.K. Bhaduri. (1971). Nucl. Phys. A 162, 289.

H.M. Nussenzveig. (1972). Causality and Dispersion Relations (Academic Press, New York.

N. Levinson, Kgl. Danski Videnskab. Selskab. (1949). Mat. Fys. 25, No. 9.

N.J. Englefield and H.S.M. Shoukry. (1974). Frog. Theor. Phys. 52, 1554.

P. Swan. (1955). Proc. Roy. Soc. A, $18,10$.

R.G. Newton. (1960). J. Math. Phys. 1, 319.

R.G. Newton. (1977). J. Math. Phys. 18, 1348.

R.G. Newton. (1977). J. Math. Phys. 18, 1582.

R. Jost and A. Pais. (1951). Phys. Rev. 82, 840.

S. Okai, S. Saito, and R. Tamagaki. (1972). Prog. Theor. Phys. 47, 484.

Special Supplement of the Progress of Theoretical Physics. (1977). No. 62, Microscopic Methods for the Interactions between Complex Nuclei" and the references therein.

S.S. Ahmed. (1974). Nuovo Cimento, 23A, 362.

S. Saito. (1968). Prog. Theor. Phys. 40, 893; 41 (1969). 705.

W. Glockle and J. Le Tourneux. (1976). Nucl. Phys. A 269, 16.

Y Singh and C.S. Warke. (1971). Can. J. Phys. 49, 1029. 8. 


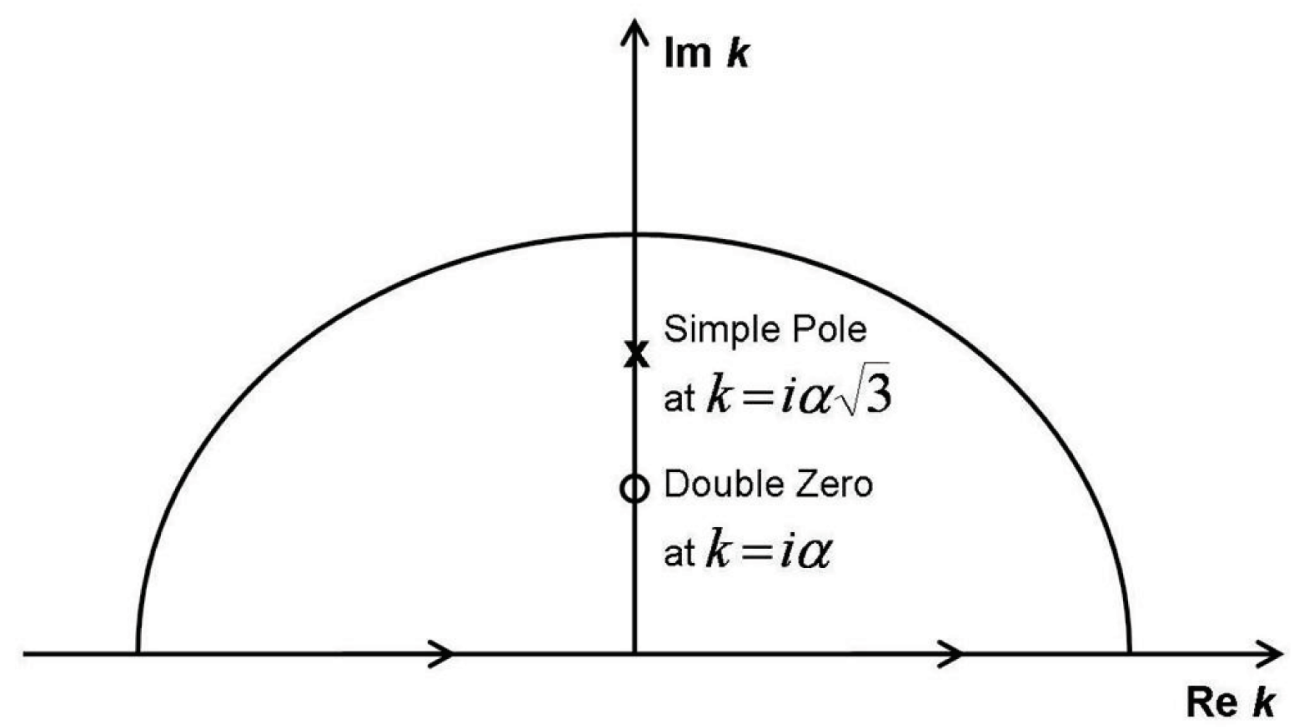

Figure 1. Poles and zeros of $\mathfrak{L}^{+}(k)$ in the upper half plane for the Saito potential. Poles are indicated by, zeros by o. There are no poles or zeros of $\mathfrak{L}^{+}(k)$ on the real axis. 